\title{
Highly Overmoded MM-wave Oscillator Experiments
}

\author{
A. W. Cross, A. J. MacLachlan, C. W. Robertson, L. Zhang, K. Ronald and A. D. R. Phelps \\ Department of Physics, SUPA, University of Strathclyde, \\ Glasgow, G4 0NG, Scotland, UK \\ a.d.r.phelps@strath.ac.uk
}

\begin{abstract}
Overmoded oscillator research has been an active area of interest at the University of Strathclyde for several years. Interaction structures based on complex electrodynamic structures can facilitate higher powers from overmoded mmwave sources. The gain media used can be vacuum electronic, or condensed matter. The present work reports on the most recent experimental results using a vacuum electronic overmoded source in W-band $(75-110 \mathrm{GHz})$. A two dimensional cylindrical slow wave interaction region is excited by an annular electron beam. Whereas in conventional sources the transverse dimensions of the slow wave structure (SWS) are comparable with the free space wavelength, in these experiments the structure diameter is approximately five free space wavelengths. Recent experimental progress is presented.
\end{abstract}

\section{INTRODUCTION}

Two dimensional periodic surface lattice (PSL) structures can provide useful advantages in the configuration of both fast-wave [1] and slow-wave [2] sources of high power electromagnetic radiation.

Research on methods of increasing the power output from vacuum electronic mm-wave sources has been actively pursued for several years at the University of Strathclyde [26]. The objective has been to increase the potential power output by increasing the cross-section of the SWS in which the electron beam transfers power to the electromagnetic wave field. A larger beam current is thereby facilitated and providing the efficiency does not decrease significantly higher mm-wave output powers are potentially attainable.

Theoretical analysis $[4,5,7,8]$ shows that by careful design of a two dimensional PSL situated on the inner surface of the cylindrical electron beam - wave interaction structure can result in a coupling of surface waves and volume waves in the interaction structure to produce a single frequency powerful output mm-wave. The concept of this coupling process has been experimentally demonstrated [9] using a planar two dimensional periodic lattice. The planar PSL can in principle be conformally mapped into a cylindrical PSL.

Initial experimental results for a $\mathrm{W}$-band slow wave overmoded oscillator using a cylindrical periodic lattice with periodicity in two dimensions and driven by an energetic electron beam were reported earlier [6] and this presentation here reports the experimental progress that has been made with a re-designed and significantly upgraded oscillator.

\section{EXPERIMENT}

A co-sinusoidal periodic corrugation, both in the axial direction and in the azimuthal direction, of the internal surface of the cylindrical SWS is predicted to give mode selectivity. Many modes can potentially be excited in a cylindrical structure of diameter $\mathrm{D}$, at a working wavelength $\lambda$, when $\mathrm{D} / \lambda \sim 5$, as in the present highly overmoded structure.

The inner surface of the cylindrical SWS, is described by equation (1)

$$
r=r_{0}+\Delta r \cos \left(k_{z} z\right) \cos (m \emptyset)
$$

where $r_{0}$ is the radius of the unperturbed waveguide, $\Delta r$ is the amplitude of the corrugation, $k_{\mathrm{z}}=2 \pi / d_{z}$, where $d_{z}$ is the period of the corrugation along the axial $\mathrm{z}$ - coordinate and $m$ is the number of azimuthal variations of the corrugation.

The present oscillator SWS has been constructed by electrodeposition of copper on a CNC machined aluminium former, that is then dissolved away using strong alkali.

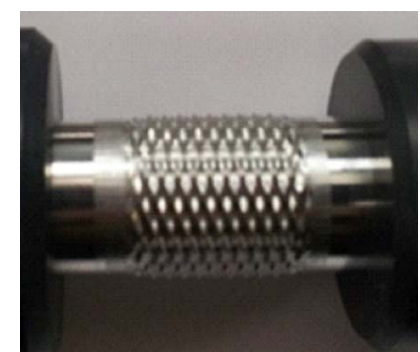

(a)

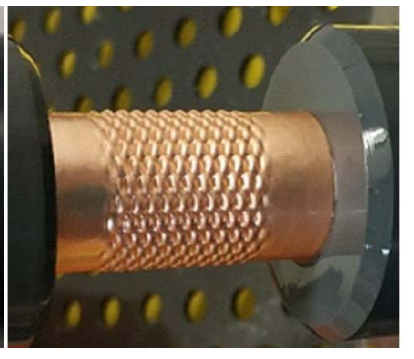

(b)
Figure 1. Method of construction of the cylindrical 2D PSL being used in the new experiment (a) aluminium former of cylindrical two dimensional SWS (b) outer view of electrodeposited copper SWS

A knife-edged annular graphite cathode, as shown in Fig. 2 , is used to produce a well-defined annular electron beam and an axially-uniform DC magnet solenoid is used to guide the electron beam through the SWS interaction structure.

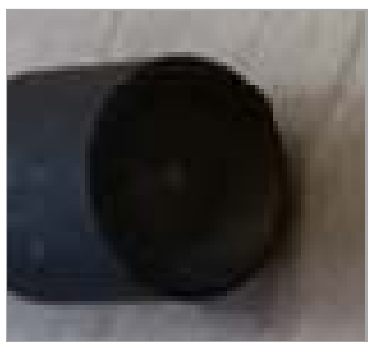

Figure 2. Annular knife-edged graphite cathode used to produce the annular electron beam in the new experiment. 
Previously [6] there was a significant perpendicular velocity component in the electron beam, whereas for the slow-wave interaction a beam with purely an axial velocity component is appropriate. In the new experiment the use of a re-designed cathode and electron gun, combined with a stronger axially uniform magnetic guiding field provides an electron beam that is better suited to producing a more efficient source than in the earlier experiment [6]. In the previous experiment a conventional water-cooled electromagnet was used to provide an axial guiding magnetic field of $2 \mathrm{~T}$, whereas in the present experiment the axial guiding magnetic field of up to $5 \mathrm{~T}$ is produced using a superconducting magnet.

In the previous experiment the output frequency was estimated by measuring the magnitude of the output signal after transmission through a set of cut-off filters. This method only provides an approximate frequency measurement and so the new experiment uses a more precise frequency measurement diagnostic. In the new experimental work a W-band local oscillator combined with a mixer and a high bandwidth oscilloscope are used in a heterodyne diagnostic to allow more precise measurement of the output frequency.

\section{NUMERICAL MODELLING}

The CST Microwave and Particle Studio software suite has been used to model the electron beam interaction with the new two dimensional PSL SWS. The parameters and dimensions of this PSL SWS configuration, the radial position of the annular electron beam, its accelerating potential and current have been used as inputs to the numerical model to simulate the present experiment. Outputs in the W-band frequency range (75-110 GHz) with over $10 \%$ electronic efficiency are predicted by the modelling, with the optimum selected 2D PSL parameters $r_{0}$, $\Delta \mathrm{r}, \mathrm{dz}, \mathrm{m}$, combined with the electron beam accelerating potentials and currents corresponding to those in the laboratory experiment.

\section{CONCLUSIONS}

Compared with the earlier experiment [6], the present experiment has a better-formed annular electron beam and the guiding solenoidal field is more uniform axially. The heterodyne frequency diagnostic enables a higher measurement precision of the output frequency to be achieved. The CST modelling shows that these highly overmoded sources demonstrate greater sensitivity to variations in the input parameters, compared with sources operating in low order modes. The use of the 2D PSL structure enables a high power stable output to be obtained. The most recent experimental measurements from this upgraded mm-wave overmoded oscillator experiment are reported.

\section{ACKNOWLEDGMENT}

David Barclay is thanked for his skilled technical assistance. Ivan Konoplev is thanked for discussions.

\section{REFERENCES}

[1] N. S. Ginzburg, N. Y. Peskov, A. S. Sergeev, et al., "The use of a hybrid resonator consisting of one-dimensional and two-dimensional Bragg reflectors for generation of spatially coherent radiation in a coaxial free-electron laser," Phys. Plasmas, vol. 9, (6), pp. 27982802, June 2002.

[2] I. V. Konoplev, et al., "Surface wave Cherenkov maser based on a periodic lattice", Appl. Phys. Lett., vol. 96, art. no. 261101, June 2010.

[3] I. V. Konoplev, et al., "Excitation of surface field cavity and coherence of electromagnetic field scattering on two-dimensional cylindrical lattice," Appl. Phys. Lett., vol.97, art. no. 261102, Dec. 2010 .

[4] I. V. Konoplev, et al., "Cylindrical, periodic surface lattice - Theory, dispersion analysis and experiment", Appl. Phys. Lett., vol. 101, art. no. 121111 , Sept. 2012.

[5] I. V. Konoplev, et al., "Cylindrical periodic surface lattice as a metadielectric: concept of a surface-field Cherenkov source of coherent radiation", Phys. Rev. A, vol. 84, art. no. 013826, July 2011.

[6] A. R. Phipps, A. J. MacLachlan, C. W. Robertson, et al., "Electron beam excitation of coherent sub-terahertz radiation in periodic structures manufactured by 3D printing," Nucl. Instrum. Methods Phys. Res. B, vol. 402, pp. 202-205, July 2017.

[7] A. J. MacLachlan, C. W. Robertson, A. W. Cross, et al., "Volume and surface mode coupling experiments in periodic surface structures for use in mm-THz high power radiation sources," AIP Advances, vol. 8, (10), art. no. 105115, Oct. 2018.

[8] A. J. MacLachlan, C. W. Robertson, I. V. Konoplev, et al., "Resonant excitation of volume and surface fields on complex electrodynamic surfaces”, Phys. Rev. Appl., vol. 11, art. no. 034034, Mar. 2019.

[9] A. J. MacLachlan, C. W. Robertson, K. Ronald, A. W. Cross, and A. D. R. Phelps, "Mode coupling in periodic surface lattice and metamaterial structures for mm-wave and $\mathrm{THz}$ applications," $\mathrm{SN}$ Appl. Sci., vol. 1, (6), art. no. 613, 2019. 